\title{
LIMIARES DE RECONHECIMENTO DE SENTENÇAS NO SILÊNCIO EM CAMPO LIVRE VERSUS LIMIARES TONAIS EM FONE EM INDIVÍDUOS COM PERDA AUDITIVA COCLEAR
}

\author{
Sentence recognition thresholds in silence in free field versus \\ pure tone thresholds in individuals with hearing loss
}

\author{
Nilvia Herondina Soares Aurélio ${ }^{(1)}$, Karine Thaís Becker ${ }^{(2)}$, Cristiane Bertolazi Padilha ${ }^{(3)}$, \\ Sinéia Neujahr dos Santos ${ }^{(4)}$, Tiago Petry ${ }^{(5)}$, Maristela Júlio Costa ${ }^{(6)}$
}

\section{RESUMO}

Objetivo: investigar a correlação existente entre os limiares tonais e os Limiares de Reconhecimento de Sentenças no Silêncio (LRSS) e verificar, se é possível, através do audiograma estabelecer um prognóstico deste paciente sobre a sua habilidade de reconhecer a fala. Métodos: foram analisados 42 indivíduos com perda auditiva coclear de grau moderado, 18 do sexo feminino e 24 do masculino, com idades entre 41 e 76 anos. Primeiramente foi realizada avaliação audiológica básica e, em seguida, a pesquisa dos Limiares de Reconhecimento de Sentenças no Silêncio, em campo livre, por meio do teste Listas de Sentenças em Português. Resultados: a análise estatística evidenciou correlação significante entre o limiar de reconhecimento de sentenças no silêncio e a média das freqüências de 0,5, 1 e $2 \mathrm{kHz}$. Por sua vez, ao correlacionar os Limiares de Reconhecimento de Sentenças no Silêncio com a média das freqüências de 3, 4 e 6 kHz, não houve correlação significante. Conclusão: o prognóstico provável da habilidade de reconhecimento de fala no silêncio, pode ser feito apenas com base nos limiares das freqüências de 0,5, 1 e $2 \mathrm{kHz}$ em perdas auditivas cocleares.

DESCRITORES: Audiologia; Audição; Percepção Auditiva; Testes de Discriminação da Fala; Audiometria de Tons Puros

(1) Fonoaudióloga do Projeto Promoção da Saúde Auditiva da Universidade Federal de Santa Maria; Mestranda em Distúrbios da Comunicação Humana pela Universidade Federal de Santa Maria.

(2) Fonoaudióloga da Prefeitura Municipal de Arroio do Meio RS; Aluna Especial do Mestrado em Distúrbios da Comunicação Humana pela Universidade Federal de Santa Maria.

(3) Fonoaudióloga da Associação dos Pais e Amigos dos Excepcionais do Município de São Borja - RS; Mestranda em Distúrbios da Comunicação Humana pela Universidade Federal de Santa Maria.

(4) Fonoaudióloga do Projeto Promoção da Saúde Auditiva da Universidade Federal de Santa Maria; Mestranda em Distúrbios da Comunicação Humana pela Universidade Federal de Santa Maria.

(5) Fonoaudiólogo do Projeto Promoção da Saúde Auditiva da Universidade Federal de Santa Maria; Mestranda em Distúrbios da Comunicação Humana pela Universidade Federal de Santa Maria.

(6) Fonoaudióloga; Professora Adjunta do Departamento de Fonoaudiologia da Universidade Federal de Santa Maria; Doutora em Ciências dos Distúrbios da Comunicação Humana pela Universidade Federal de São Paulo.

\section{INTRODUÇÃO}

A audição possibilita o desenvolvimento das nossas capacidades comunicativas desde cedo ${ }^{1}$, sendo a habilidade para reconhecer a fala, um de seus aspectos mais importantes ${ }^{2}$. Por ser uma habilidade tão significante para o indivíduo é que uma das queixas mais comuns na rotina clínica seja, justamente, a dificuldade de compreender a fala ${ }^{3}$. Sendo assim, a habilidade de compreensão da fala deve ser considerada o aspecto mais importante a ser mensurado na função auditiva humana, pois permite avaliar a função comunicativa receptiva, fornecendo dados de como o indivíduo se comporta em situações de escuta diária, por meio de informações objetivas, facilmente quantificáveis ${ }^{4}$.

Em uma avaliação audiológica, as dificuldades na compreensão da fala só podem ser realmente evidenciadas com estímulos de fala que representem uma situação comunicativa ${ }^{5}$. Testes audiológicos 
que empregam sentenças como estímulo, oferecem uma aproximação direta com situações de comunicação do dia-a-dia e fornecem informações que vão orientar a conduta mais adequada a ser indicada para o indivíduo com queixa de distúrbio da comunicação, além de avaliar a real habilidade auditiva do paciente ${ }^{6}$. Na avaliação em campo livre, o material e a situação de teste permitem avaliar a situação binaural. Tal situação reflete a condição na qual a comunicação diária se estabelece ${ }^{7}$.

O teste Listas de Sentenças em Português (LSP), foi elaborado por Costa em 1998, utilizando frases como estímulo, que podem ser aplicadas em situações de silêncio e na presença de ruído competitivo ${ }^{6}$. O desempenho do indivíduo neste teste fornecerá informações sobre a real habilidade de reconhecimento da mensagem falada, sendo que este dependerá do tipo e grau da perda auditiva ${ }^{6}$.

A perda auditiva coclear ocorre quando o aparelho de transmissão do som encontra-se normal, mas há uma alteração na qualidade do som (lesões da orelha interna ou do órgão de Corti) ${ }^{8}$. A discriminação é afetada em todos os casos. Pelo comprometimento das células sensoriais da orelha interna e, dependendo de sua gravidade, a inteligibilidade das palavras é afetada e pior será quanto maior for a perda auditiva. $O$ efeito sobre a discriminação será menor se as freqüências da fala $(0,25 \mathrm{a} 2 \mathrm{kHz})$ forem menos comprometidas. Em perdas atingindo apenas freqüências mais altas (acima de $2 \mathrm{kHz}$ ), a discriminação poderá ser pouco comprometida ${ }^{8}$. A classificação das perdas auditivas quanto ao grau é baseada na média dos limiares da via aérea nas freqüências de $500,1.000$ e $2.000 \mathrm{~Hz}$, sendo que a perda auditiva de grau moderado é caracterizada pela média tritonal de 41 a $70 \mathrm{~dB}$ (Davis \& Silverman, 1970) ${ }^{9}$.

Na prática clínica, é comum encontrar-se sujeitos com grau e configuração de perda auditiva neurossensorial semelhantes, que apresentam habilidades substancialmente diferentes quanto à percepção de fala. Existe uma relação relativamente pobre entre limiares auditivos tonais e inteligibilidade de fala para indivíduos portadores da perda auditiva neurossensorial. Provavelmente, além da sensibilidade auditiva, outros fatores interferem na percepção de fala $^{2}$. Isto justifica a necessidade de investigar se é possível estabelecer um prognóstico do indivíduo com perda auditiva coclear moderada, no que se refere à habilidade de reconhecimento de fala e conseqüente capacidade de comunicação, a partir dos limiares tonais. Pela análise destes dados pode-se também obter mais informações para orientar um candidato ao uso de próteses auditivas.

Baseado nestas considerações, o presente estudo tem por objetivo: verificar a correlação exis- tente entre os limiares tonais obtidos em fone na rotina audiológica e os Limiares de Reconhecimento de Sentenças no Silêncio (LRSS) em campo livre, em pacientes com perda auditiva coclear de grau moderado; e, verificar se é possível, por meio do audiograma estabelecer um prognóstico deste paciente sobre a sua habilidade de comunicação.

\section{MÉTODOS}

O presente estudo foi realizado no Ambulatório de Audiologia do Serviço de Atendimento Fonoaudiológico (SAF) da Universidade Federal de Santa Maria (UFSM), a partir do Projeto "Pesquisa e base de dados em saúde auditiva" registrado no GAP do Centro de Ciências da Saúde sob o no 019731.

Para constituir o grupo de estudo, foram selecionados pacientes do Laboratório de Próteses Auditivas do Serviço de Atendimento Fonoaudiológico (SAF) da Universidade Federal de Santa Maria, atendidos no período de novembro e dezembro de 2006 e março e abril de 2007 , constituindo um total de 42 indivíduos, 18 do sexo feminino e 24 do sexo masculino, com idades entre 41 e 76 anos, com a ATL característica de perda auditiva coclear de grau moderado, ou seja, ausência de gap entre os limiares das vias aéreas e ósseas no mesmo ouvido, ou este, não superior a $10 \mathrm{~dB}$ NA e média das freqüências da fala $(0,5,1$ e $2 \mathrm{kHz})$ entre $41 \mathrm{~dB}$ NA e $70 \mathrm{~dB}$ NA (Davis \& Silverman, 1970) ${ }^{9}$.

Foram observados como critérios de exclusão a presença de alterações neurológicas, articulatórias e/ou de fluência verbal; a presença de rolha de cerúmen ou de outras alterações no meato acústico externo capazes de alterar o desempenho no teste; ausência de resposta ao teste Listas de Sentenças em Português e dificuldade para memorizar as sentenças.

Os voluntários receberam orientações sobre os objetivos, justificativa e metodologia do estudo proposto e assinaram o Termo de Consentimento Livre e Esclarecido. Após, os indivíduos foram submetidos inicialmente a anamnese, e em seguida a meatoscopia e a avaliação audiológica básica, da qual fizeram parte:

- Audiometria Tonal Liminar (ATL): por via aérea nas freqüências de 250 a $8000 \mathrm{~Hz}$ e por via óssea nas freqüências de 500 a 4000 Hz;

- Pesquisa do Limiar de Reconhecimento de Fala (LRF), com palavras dissilábicas apresentadas à viva-voz;

- Pesquisa do Índice Percentual de Reconhecimento de Fala (IPRF), com palavras monossilábicas apresentadas à viva-voz.

Para a obtenção dos dados da pesquisa foi utilizado um audiômetro digital de dois canais, marca 
Fonix, modelo FA-12, tipo I, (norma de calibração IEC 645-2: 1993) e um sistema de amplificação para audiometria em campo livre, alimentação 110/220 Volts Ac, $50 / 60 \mathrm{~Hz}$, potência de saída do amplificador 80 watts, potência das caixas 100 watts.

Após a avaliação audiológica básica, foi realizada a pesquisa dos Limiares de Reconhecimento de Sentenças no Silêncio (LRSS), por aplicação do teste Listas de Sentenças em Português (LSP), apresentado por Costa (1998). Esse material apresenta-se gravado em CD e contém listas de sentenças em português e um ruído com espectro de fala, gravados em canais independentes, permitindo a apresentação das sentenças tanto no silêncio, quanto no ruído, com intensidades de apresentação diferentes. As sentenças foram apresentadas utilizando-se um Compact Disc Player Digital Toshiba 4149, acoplado ao audiômetro acima descrito.

As sentenças foram apresentadas em campo livre, permitindo avaliar as duas orelhas simultaneamente. As listas de sentenças utilizadas na pesquisa estão em anexo (Figura 1).

A apresentação do material de teste (LSP) aos participantes obedeceu à seguinte ordem: a) Apresentação das sentenças de 1 a 10 da lista $1 \mathrm{~A}$, em campo livre, no silêncio para familiarização do indivíduo com o teste;

b) Apresentação da lista 1B em campo livre, no silêncio.

Para estabelecer a intensidade de apresentação das sentenças, foi usado como referência um tom puro gravado no canal do CD onde estão gravadas as sentenças, pois o sinal de fala é um som complexo, que apresenta uma variação de $30 \mathrm{~dB}$ entre o som mais intenso e o menos intenso, oscilando $12 \mathrm{~dB}$ acima e $18 \mathrm{~dB}$ abaixo da média ${ }^{10}$, necessitando, portanto de um som contínuo de referência para manter sempre as mesmas condições de apresentação.

A saída de cada canal do CD foi calibrada através do VU-meter do audiômetro antes do início da avaliação de cada indivíduo. O tom de um $\mathrm{kHz}$ presente no mesmo canal do CD em que estão gravadas as sentenças foi colocado no nível zero.

Todo o procedimento foi realizado em uma cabina acusticamente tratada, com o paciente posicionado de frente, a um metro em relação à caixa acústica, com o sinal de fala (sentenças) sendo sempre

\section{Lista $1 \mathrm{~A}$}

1. Não posso perder o ônibus.

2. Vamos tomar um cafezinho.

3. Preciso ir ao médico.

4. A porta da frente está aberta.

5. A comida tinha muito sal.

6. Cheguei atrasado para a reunião.

7. Vamos conversar lá na sala.

8. Depois, liga para mim.

9. Esqueci de pagar a conta.

10. Os preços subiram ontem.

11. O jantar está na mesa.

12. As crianças estão brincando.

13. Choveu muito nesse fim-de-semana.

14. Estou morrendo de saudade.

15. Olhe bem ao atravessar a rua.

16. Preciso pensar com calma.

17. Guardei o livro na primeira gaveta.

18. Hoje é meu dia de sorte.

19. O sol está muito quente.

20. Sua mãe acabou de sair de carro.

21. Ela vai viajar nas férias.

22. Não quero perder o avião.

23. Eu não conheci sua filha.

24. Ela precisa esperar na fila.

25. O banco fechou sua conta

\section{Lista 1B}

1. O avião já está atrasado.

2. O preço da roupa não subiu.

3. O jantar da sua mãe estava bom.

4. Esqueci de ir ao banco.

5. Ganhei um carro azul lindo.

6. Ela não está com muita pressa.

7. Avisei seu filho agora.

8. Tem que esperar na fila.

9. Elas foram almoçar mais tarde.

10. Não pude chegar na hora. 
apresentado a $0^{\circ}$ Azimute, ou seja, formando um ângulo de $0^{\circ}$ em relação aos planos horizontal e vertical. Essa posição é a mais vantajosa, pois permite maior precisão nas medidas e conforto ao paciente, além de ser a que mais se aproxima de uma situação de conversação normal ${ }^{11}$.

Foi realizada a calibração do equipamento em função das características do sinal de teste e das condições acústicas do ambiente, antes de iniciar as medidas em campo livre, estabelecendo, assim, os níveis de pressão sonora, em que a fala era percebida na orelha do indivíduo testado. As medidas foram obtidas com o auxílio de um Medidor de Pressão Sonora Digital, Marca Radio Shack, posicionado em um ponto médio entre as duas orelhas, a uma distância de um metro do alto-falante.

A escala utilizada foi a Escala A, com respostas rápidas, por ser considerada a que melhor se aproxima da resposta humana, além de ser a mais usada pela maioria dos pesquisadores nesta área ${ }^{3,7,11,12}$.

Todos os indivíduos ao iniciar o teste foram treinados com a lista $1 \mathrm{~A}$, que consiste em uma lista de 25 sentenças. O treinamento foi realizado apresentando no silêncio as 10 primeiras sentenças da lista $1 \mathrm{~A}$ na condição de escuta binaural.

A intensidade inicial de apresentação da primeira sentença de cada lista do teste, propriamente dito, foi baseada nos resultados encontrados no treinamento acima descrito. Por meio desse treinamento, foi possível estabelecer para cada indivíduo o nível de intensidade necessária para que este tivesse êxito na primeira sentença de cada lista do teste.

Quando a resposta era correta, diminuía-se a intensidade de apresentação do estímulo seguinte. Quando a resposta era incorreta, aumentava-se a intensidade de apresentação do estímulo seguinte. Uma resposta só foi considerada correta, quando o indivíduo repetiu, sem nenhum erro ou omissão, toda a sentença apresentada.

A literatura sugere que sejam utilizados intervalos de $4 \mathrm{~dB}$ até a primeira mudança no tipo de resposta e, posteriormente, os intervalos de apresentação dos estímulos sejam de $2 \mathrm{~dB}$ entre si até o final da lista ${ }^{13}$. Entretanto, devido às possibilidades técnicas do equipamento disponível para a realização desta pesquisa, foram utilizados intervalos de apresentação das sentenças de $5 \mathrm{~dB}$ e 2,5 $\mathrm{dB}$, respectivamente.

A média dos valores foi calculada a partir do nível de apresentação em que ocorreu a primeira mudança no tipo de resposta, até o valor de apresentação da última sentença da lista.

Após a pesquisa dos limiares tonais e do LRSS, foram feitas as médias tritonais, com os dados da melhor orelha (pois em uma avaliação em campo livre é esta quem mais participa do resultado) nas freqüências de 0,5, 1 e $2 \mathrm{kHz}$ e também 3, 4 e $6 \mathrm{kHz}$.

Este estudo foi aprovado pela Comissão de Ética em Pesquisa (CEP) da UFSM em 05/12/2006, protocolo no 23081.0.016862/2006-09.

Com relação aos métodos estatísticos utilizados, para analisar a distribuição do grupo de estudo foi utilizado o Chi-Square test. É um teste não paramétrico, cujo princípio básico é comparar proporções, isto é, as possíveis divergências entre as freqüências observadas e esperadas para um certo evento ${ }^{14}$.

Para investigar o nível de correlação entre os resultados obtidos na pesquisa do LRSS e a média dos limiares tonais para as freqüências de 0,5, 1 e $2 \mathrm{kHz}$ e 3, 4 e $6 \mathrm{kHz}$ foi aplicado o Coeficiente de Correlação de Pearson. O nível de rejeição para a hipótese de nulidade foi fixado em um valor menor ou igual a $0,05(5 \%)$.

\section{RESULTADOS}

Inicialmente, os resultados obtidos sobre a distribuição do grupo de indivíduos estudados, foram analisados (considerando as variáveis sexo e idade) por meio do teste Chi-Square, o que evidenciou não haver diferença estatisticamente significante entre os sexos, assim como, entre adultos e idosos.

$\mathrm{Na}$ Tabela 1, estão expostas as médias aritméticas, os desvios padrão e os valores mínimo e máximo referentes aos limiares de reconhecimento de sentenças no silêncio (LRSS) e à média dos limiares tonais da melhor orelha para as freqüências de 0,5, 1 e $2 \mathrm{kHz}$ e 3,4 e $6 \mathrm{kHz}$ obtidos dos 42 indivíduos avaliados.

Tabela 1 - Médias aritméticas, desvios padrão e valores mínimo e máximo referentes ao LRSS e limiares tonais $n=42$

\begin{tabular}{ccccc}
\hline VARIÁVEL & MÉDIA & DESVIOPADRÃO & MÍNIMO & MÁXIMO \\
\hline LRSS & $64,48 \mathrm{~dB} \mathrm{~A}$ & $5,21 \mathrm{~dB} A$ & $46,80 \mathrm{~dB} \mathrm{~A}$ & $71,30 \mathrm{~dB} A$ \\
Média de 0,5, 1 e 2 kHz & $49,05 \mathrm{~dB}$ NA & $5,91 \mathrm{~dB}$ NA & $41,70 \mathrm{~dB}$ NA & $63,30 \mathrm{~dB}$ NA \\
Média de 3, 4 e $6 \mathrm{kHz}$ & $64,44 \mathrm{~dB}$ NA & $8,43 \mathrm{~dB}$ NA & $51,20 \mathrm{~dB} \mathrm{NA}$ & $82,50 \mathrm{~dB}$ NA \\
\hline
\end{tabular}

Legenda: LRSS - Limiar de Reconhecimento de Sentenças no Silêncio 
Tabela 2 - Correlação do LRSS (dB A) com a média dos limiares tonais (dB NA) de 0,5, 1 e 2 $\mathrm{kHz}(\mathrm{n}=42)$

\begin{tabular}{ccc}
\hline Estatísticas & LRSS & $\begin{array}{c}\text { Média 0,5, } \\
\text { e e } 2 \mathrm{kHz}\end{array}$ \\
\hline Média & 64,47 & 49,05 \\
\hline $\begin{array}{c}\text { Coeficiente de } \\
\text { correlação }\end{array}$ & $\mathrm{r}=0,51157^{\star}$ \\
\hline
\end{tabular}

$\left({ }^{*}\right)$ Existe correlação estatisticamente significante $(p<0,05)$ Legenda: LRSS - Limiar de Reconhecimento de Sentenças no Silêncio; Teste estatístico utilizado: Coeficiente de Correlação de Pearson

Tabela 3 - Correlação do LRSS (dB A) com a média dos limiares tonais (dB NA) de 3, 4 e 6 $\mathrm{kHz}(\mathrm{n}=42)$

\begin{tabular}{ccc}
\hline Estatísticas & LRSS & $\begin{array}{c}\text { Média 3, } \\
4 \text { e } 6 \mathrm{kHz}\end{array}$ \\
\hline Média & 64,47 & 64,44 \\
\hline $\begin{array}{c}\text { Coeficiente de } \\
\text { correlação }\end{array}$ & \multicolumn{2}{r}{$=0.13104$} \\
\hline
\end{tabular}

$\left({ }^{*}\right)$ Existe correlação estatisticamente significante $(p<0,05)$ Legenda: LRSS- Limiar de Reconhecimento de Sentenças no Silêncio; Teste estatístico utilizado: Coeficiente de Correlação de Pearson

Nas Tabelas 2 e 3, estão expostos os resultados da análise de correlação entre o limiar de reconhecimento de sentenças no silêncio (LRSS) e as médias dos limiares tonais para as freqüências de 0,5, 1 e $2 \mathrm{kHz}$ e 3, 4 e $6 \mathrm{kHz}$, respectivamente, obtida para os 42 indivíduos, a partir da aplicação do Coeficiente de Correlação de Pearson. Os resultados estatisticamente significantes foram assinalados com um asterisco $\left(^{*}\right)$.

\section{DISCUSSÃo}

Ao analisar a correlação entre o limiar de reconhecimento de sentenças no silêncio e a média das freqüências de 0,5, 1 e $2 \mathrm{kHz}$, utilizando o teste Listas de Sentenças em Português (LSP) ${ }^{6}$, verificou-se que o LRSS médio obtido na avaliação dos 42 indivíduos foi $64,48 \mathrm{~dB}$ A e a média dos limiares tonais para as freqüências de $0,5,1$ e $2 \mathrm{kHz}$ foi de 49,05 dB NA (Tabela 1). Ao correlacionar estes dados, a análise estatística revelou que existe correlação estatisticamente significante entre as variáveis $(r=0,51157)$. O grau de correlação foi considerado moderado (Tabela 2 ).

Os resultados desta pesquisa vão ao encontro dos obtidos por outros pesquisadores que igual- mente realizaram uma análise comparativa do valor médio dos LRSS com a média dos limiares tonais de $0,5,1$ e $2 \mathrm{kHz}$, afirmando que essas duas variáveis estão intimamente relacionadas. Em um estudo com 100 indivíduos normo-ouvintes, os autores referiram que a média dos limiares tonais nas freqüências de 0,5, 1 e $2 \mathrm{kHz}$ pode ser usada como referência para a análise dos resultados obtidos na pesquisa do LRSS ${ }^{4}$.

Demais autores ${ }^{15-17}$ ressaltaram o fato de que a média de 0,5, 1 e $2 \mathrm{kHz}$ teria boa relação com a previsão do LRSS e ainda acrescentaram que o único parâmetro que influencia o LRSS é o limiar de audibilidade. Pelo fato das sentenças fornecerem pistas acústicas e lingüísticas com significado, pode-se esperar também, que os valores dos LRSS sejam melhores que os valores obtidos a partir das médias dos limiares tonais de 0,5, 1 e $2 \mathrm{kHz}{ }^{4}$.

Por sua vez, ao correlacionar o LRSS com a média das freqüências de 3 , 4 e $6 \mathrm{kHz}$, não houve correlação significante $(r=0,13104)$ (Tabela 3), o que não quer dizer que estas freqüências não influenciem o reconhecimento de fala do indivíduo, mas sim que há uma grande dispersão entre as respostas, mostrando que em situações silenciosas, há uma série de fatores individuais a serem considerados alem da audibilidade das freqüências de 3 , 4 e 6 kHz. Deve-se levar em consideração a influência da inteligência, memória e do conhecimento do indivíduo avaliado sobre o resultado dos testes que utilizam sentenças como estímulo de fala ${ }^{18}$. Vários fatores que podem interferir nas respostas de avaliação de reconhecimento de fala, como a motivação, o interesse, o nível intelectual e educacional, a idade, a familiaridade com as palavras e o nível de estresse do paciente ${ }^{2}$.

Esses resultados mostram que ao analisar um audiograma para tentar estabelecer um prognóstico da habilidade de comunicação deste indivíduo, sem a utilização de testes de fala, apenas pode-se afirmar que o indivíduo terá respostas correlacionadas com as freqüências de 0,5, 1 e $2 \mathrm{kHz}$, em situação silenciosa, mas mesmo assim de forma moderada, como mostram os índices de correlação.

Muitos audiologistas e clínicos referem-se às freqüências de 0,5, 1 e $2 \mathrm{kHz}$ como "freqüências da fala", levando ao falso conceito de que as freqüências acima de $2 \mathrm{kHz}$ e abaixo de $0,5 \mathrm{kHz}$ não sejam importantes para o reconhecimento de fala. As freqüências acima de $3 \mathrm{kHz}$ são tão importantes para o reconhecimento da fala quanto as freqüências abaixo de $1 \mathrm{kHz}$. Pesquisas também indicam que as freqüências entre 4 e $6 \mathrm{KHz}$ são importantes para o reconhecimento de consoantes ${ }^{19}$.

A maior parte dos sujeitos com perda auditiva nas freqüências altas (acima de $3 \mathrm{KHz}$ ) pode referir 
pouca ou nenhuma dificuldade em compreender a fala em ambientes silenciosos, já que nestas situações existe uma série de pistas excedentes que os mesmos podem utilizar para compreender a fala. Entretanto, em ambiente ruidoso ou em situações adversas, o sujeito pode apresentar inúmeras dificuldades na inteligibilidade da fala, pois o número de pistas cai significativamente, levando-os a utilizar somente pistas disponíveis na situação ${ }^{\text {? }}$.

Assim, ressalta-se a importância da realização de testes na presença de ruído, já que pacientes com as mesmas habilidades de reconhecimento de fala no silêncio podem apresentar resultados extremamente diferentes em ambientes ruidosos.

Deve-se ter a preocupação em medir a capacidade de discriminação da fala em situações mais próximas da vida real, nas quais o indivíduo está exposto a uma série de estímulos discriminativos concorrentes ${ }^{6}$. Todo o paciente com queixa de dificuldade em compreender a fala deveria ser submetido a uma avaliação de reconhecimento de fala no silêncio e na presença de ruído de fundo ${ }^{20}$.

É de grande importância salientar, que as Desordens do Processamento Auditivo (DPA), que são definidas pela deficiência de uma ou mais áreas dos fenômenos comportamentais auditivos como localização sonora, discriminação sonora, reconhecimento auditivo, aspectos temporais da audição (resolução, mascaramento, integração e seqüência temporal), desempenho auditivo com sinais acústicos em competição e desempenho auditivo em situações acústicas desfavoráveis podem influen- ciar de maneira negativa nos achados de testes de reconhecimento de fala ${ }^{18}$. O que justifica a necessidade de aplicar testes que avaliem o processamento auditivo em indivíduos com queixa de dificuldade de compreensão da fala.

Este estudo permite sugerir, portanto que, se o audiologista quer saber como é a condição real de comunicação de um indivíduo, deve introduzir na rotina clínica, testes que utilizem sentenças como estímulo, porém para que esta avaliação seja completa e mostre realmente como este indivíduo vai lidar com todas as situações de comunicação do dia-a-dia, o ideal é aplicar testes que avaliem o processamento auditivo, além de testes que utilizem sentenças na presença de ruído.

\section{CONCLUSÃO}

Ao término desta pesquisa, a análise crítica dos resultados obtidos para o grupo estudado permitiu concluir que nos indivíduos com perda auditiva coclear de grau moderado houve correlação entre a média dos limiares tonais de 0,5, 1 e $2 \mathrm{kHz}$ e o LRSS realizados em campo livre. Por outro lado, não houve correlação entre a média dos limiares tonais de 3,4 e $6 \mathrm{kHz}$ e o LRSS realizados em campo livre. Podendo-se inferir que, para o grupo de indivíduos estudados, o prognóstico provável da habilidade de reconhecimento de fala no silêncio, pode ser feito apenas com base nos limiares das freqüências de $0,5,1$ e $2 \mathrm{kHz}$.

\section{ABSTRACT}

Purpose: to investigate the existent correlation among pure tone thresholds and Sentence Recognition Thresholds in Silence (TRSS) and to check if it is possible, through the audiogram, to set up a prognostic of the patients about their communication ability. Methods: 42 individuals with moderate cochlear hearing loss, 18 females and 24 males, 41 to 76 - year old were studied. Firstly, a basic audiologic evaluation was carried out, then a search of TRSS in free field, through the Portuguese Sentence List Test (PSLT) (Costa, 1998). Results: The statistical analysis showed significant correlation between the sentence recognition thresholds in silence and the average of frequencies of $0.5,1$ and $2 \mathrm{kHz}$. However, when correlating with the average frequencies of 3,4 e $6 \mathrm{kHz}$, there was no significant correlation. Conclusion: the probable prognostic concerning the ability for recognizing sentences in silence can be carried through just with the frequency thresholds of $0.5,1$ and $2 \mathrm{kHz}$ in cochlear hearing loss.

KEYWORDS: Audiology; Hearing; Auditory Perception; Speech Discrimination Tests; Audiometry, Pure-Tone 


\section{REFERÊNCIAS}

1. Gatto $\mathrm{Cl}$, Tochetto TM. Deficiência auditiva infantil: implicações e soluções. Rev CEFAC. 2007; 9(1):110-5.

2. Caporali SA, Silva JA. Reconhecimento de fala no ruído em jovens e idosos com perda auditiva. Rev Bras Otorrinolaringol. 2004; 70(4):525-32.

3. Miranda EC, Costa MJ. Reconhecimento de sentenças no silêncio e no ruído de indivíduos jovens adultos normo-ouvintes em campo livre. Fono Atual. 2006; 8(35):4-12.

4. Soncini F, Costa MJ, Oliveira TMT, Lopes LFD. Correlação entre limiares de reconhecimento de sentenças no silêncio e limiares tonais. Rev Bras Otorrinolaringol. 2003; 69(5):672-7.

5. Freitas CD, Lopes LFD, Costa MJ. Confiabilidade dos limiares de reconhecimento de sentenças no silêncio e no ruído. Rev Bras Otorrinolaringol. 2005; 71(5):624-32.

6. Costa MJ. Lista de sentenças em português: apresentação e estratégias de aplicação na audiologia. Santa Maria: Pallotti; 1998. p. 44.

7. Henriques MO, Miranda EC, Costa MJ. Limiares de reconhecimento de sentenças no ruído, em campo livre: valores de referência para adultos normo-ouvintes. Rev Bras Otorrinolaringol. 2008; 74(2):188-92.

8. Lopes Filho O. Deficiência auditiva. In: Lopes Filho O. Tratado de fonoaudiologia. São Paulo: Roca; 1997. p. 3-24.

9. Davis H, Silverman SR. Interpretação dos resultados da avaliação audiológica. In: Santos TMM, Russo ICP. A prática da audiologia clínica. São Paulo: Cortez; 1991. p.175-96.

10. Boothroyd A. Speech perception, sensorioneural hearing loss and hearing aid. In: Studevaker G,
Hochberg I. Acustical factors affevting hearing aid performance. Boston: Allyn \& Bacon; 1993. p. 277-99.

11. Henriques MO, Costa MJ. Limiares de reconhecimento de sentenças, em campo livre, na presença de ruído incidente de diferentes ângulos. Fono Atual. 2006; 38(7):12-24.

12. Mendel LL. Objective and subjective hearing aid assessment outcomes. Am J Audiol. 2007; 16(2):118-29.

13. Levitt $H$, Rabiner LR. Use of a sequencial strategy in intelligibility testing. J Acoust Soc Am. 1967; 42:609-12.

14. Weisstein EW. Making mathWorld. The Matematica Journal. 2007;10(3):474-88.

15. Smoorenburg GF. Speech reception in quiet and in noise conditions by individuals with noise-induced hearing loss in relation to their tone audiogram. J Acoust Soc Am. 1992; 91(1):421-37.

16. Plomp R. Auditory handicap of hearing impairment and the limited benefit of hearing aids. J Acoust Soc Am. 1978; 63(2):533-49.

17. Wong LL, Cheung C, Wong EC. Comparison of hearing thresholds obtained using pure-tone behavioral audiometry, the Cantonese Hearing in Noise Test (CHINT) and cortical evoked response audiometry. Acta Otolaryngol. 2008; 128(6):654-60. 18. Neves VT, Feitosa MAG. Controvérsias ou complexidade na relação entre processamento temporal auditivo e envelhecimento?. Rev Bras Otorrinolaringol. 2003; 69(2):1-11.

19. Wilson $\mathrm{RH}$, Strouse AL. Audiometria com estímulos de fala. In: Musiek FE, Rintelmann NF. Perspectivas atuais em avaliação auditiva. São Paulo: Manole; 2001. p. 21-54.

20. Cooper JC, Cutts BP. Speech discrimination in noise. J Speech Hear Res. 1971; 14(2):332-7.

RECEBIDO EM: 08/01/2008

ACEITO EM: 24/06/2008

Endereço para correspondência:

Rua Venâncio Aires, 454-A

Santa Maria - RS

CEP: 97010-001

Tel: (55) 3226-9238 / (55) 8414-3357

E-mail: nilviaaurelio@yahoo.com.br 